To link to this article / Para enlazar con este artículo:

https://doi.org/10.14198/fem.2020.35.10

To cite this article / Para citar este artículo:

Johnson, María Cecilia. «Las TRHA y los debates en la academia feminista sobre reproducción: relaciones de poder y tecnología». En Feminismo/s, 35 (junio 2020): 263-289. DOI: 10.14198/fem.2020.35.10

\title{
LAS TRHA Y LOS DEBATES EN LA ACADEMIA FEMINISTA SOBRE REPRODUCCIÓN: RELACIONES DE PODER Y TECNOLOGÍA
}

\author{
ARTS AND THE DEBATES IN THE FEMINIST \\ REPRODUCTION ACADEMY: POWER RELATIONS AND \\ TECHNOLOGY
}

\author{
María Cecilia JOHNSON \\ CIECS-CONICET. Universidad Nacional de Córdoba, Córdoba (Argentina) \\ cecilia.johnson@unc.edu.ar \\ orcid.org/0000-0002-5102-2504
}

\section{Resumen}

Este trabajo tiene como objetivo reconstruir los debates que desde la academia y los activismos feministas se han planteado sobre la reproducción humana asistida. Mediante un recorrido histórico se abordan estos debates que comienzan en el norte global, desde los debates de la segunda ola, los feminismos queer y los desafíos para los feminismos en Latinoamérica. Se advierte una tensión sobre algunos ejes centrales para el feminismo: desde la pregunta por las nuevas formas de opresión sobre los cuerpos, hasta quiénes debaten por las posibilidades de agencia y autonomía reproductiva. Estas tensiones adquieren matices diferentes que no pueden disociarse de las diferentes posturas epistemológicas sobre cuerpo, autonomía y poder que proponen, así como tampoco se pueden separar de los escenarios globales y regionales que suponen entender el trabajo reproductivo de forma estratificada.

Palabras clave: feminismos; reproducción asistida; poder; autonomía; América Latina. 


\begin{abstract}
This work aims to reconstruct the debates from the academy and feminist activists have been raised about assisted human reproduction. From a historical point of view, these debates that begin in the global north are addressed, from the debates of the second wave feminism, queer feminisms and the challenges for feminisms in Latin America. A tension is noticed on some central axes for feminism: from the question about the new forms of oppression on the bodies to those who debate the possibilities of agency and reproductive autonomy. These tensions acquire different nuances that cannot be separated from the different epistemological positions on body, autonomy, and power they propose, nor can they be separated from the global and regional scenarios that involve understanding reproductive work in a stratified way.
\end{abstract}

Keywords: Feminisms; Assisted reproduction; Power; Autonomy; Latin America.

\title{
1. INTRODUCCIÓN
}

El estudio de las experiencias de personas usuarias de la medicina reproductiva no siempre fue abordado por el feminismo de la misma manera. Podría pensarse que desde los años setenta las Técnicas de Reproducción Humana Asistida (TRHA) han reactivado algunos debates históricos dentro del feminismo, así también han planteado nuevos interrogantes fuera de este campo.

En el marco de una investigación doctoral en Estudios de Género que estudia las experiencias de mujeres usuarias de reproducción asistida en Argentina, este trabajo se propone como objetivo principal reconstruir algunos debates en clave historiográfica dentro de la academia y los activismos feministas sobre las TRHA. Ello supone identificar en primera instancia algunos nudos críticos de discusión dentro de este movimiento y sobre el que continúan existiendo desacuerdos y tensiones entre posiciones epistémicas y geopolíticas al pensar la reproducción. La maternidad constituye un primer nudo crítico de discusión, sobre el que han existido miradas contrapuestas en los feminismos. Se identifican dentro de una compleja discusión dos grandes posturas que podrían resumirse entre aquellos sectores que ponen el acento en su carácter opresivo de la maternidad y entre quienes consideran que las opresiones son producto de una sociedad patriarcal. De este planteamiento se desprende el interrogante sobre las posibilidades de construir autonomía sexual y reproductiva: ¿es posible pensar la autonomía reproductiva

Feminismo/s 35, junio 2020, pp. 263-289 
solamente cuando se elige no maternar o también se construye autonomía cuando se elige la maternidad?

Un segundo nudo que atraviesa de forma transversal estas discusiones en los estudios feministas es la pregunta por el sujeto del feminismo, encabezado por las posiciones críticas feministas en ruptura con el feminismo blanco, universal y heterosexual, posición cuestionada desde otras intersecciones.

Reconocer estos nudos críticos permite comprender la genealogía de ciertas discusiones en los feminismos, las cuales se reeditan cuando hablamos de las TRHA. De esta manera la pregunta por la maternidad y poder se traduce en la pregunta por la tecnología reproductiva y el poder: ¿La tecnología reproductiva constituye una forma de opresión patriarcal o puede ser pensada como fuente de liberación de las mujeres en su reproducción?

Para desarrollar estas discusiones luego de esta introducción -sección primera-, este artículo desarrolla las diferentes posiciones en el debate. En una segunda sección se recuperan las diversas posiciones que desde la teoría y el activismo feministas han pensado críticamente la maternidad y las tecnologías reproductivas. Este análisis supone un paralelo con las posiciones y aportes de la teoría feminista sobre la maternidad que permiten adentrarse al pensamiento y crítica feministas sobre las TRHA. Así mismo estos interrogantes permiten presentar las primeras fracciones dentro del feminismo sobre las TRHA.

En una tercera sección se desarrollan los debates que fueron atravesando los feminismos de la segunda ola del norte global, y la discusión con otras posiciones del feminismo queer de los años noventa y contemporáneos que plantean otra relación con la tecnología como dispositivo de poder.

Los debates presentes en estas secciones dan cuenta de un primer antagonismo: para algunas feministas radicales, las TRHA fueron identificadas como una forma más de opresión patriarcal sobre los cuerpos de las mujeres, mientras que desde otras posiciones se plantearon distintas maneras de apropiación, resistencia y hasta consideraron a las TRHA como una plataforma de liberación utópica.

El desarrollo de los estudios de la sexualidad y de la biopolítica modificó la mirada sobre las THRA, ahora entendidas como parte del creciente proceso de control biopolítico y medicalización de los cuerpos (Armstrong 393; Foucault 17; Rose 10). Así mismo, el desarrollo de estudios feministas que 
comienzan a disputar el campo de la salud y las experiencias de usuarias en el campo biomédico generó nuevas discusiones. Más aún, otros sectores del feminismo encuentran en la tecnología reproductiva una fuente utópica de liberación o de subversión del género.

En una cuarta sección, se retoman algunas producciones de Argentina que discuten sobre las TRHA y los debates situados desde los feminismos del sur y decoloniales como otro espacio situado para pensar estas intersecciones, donde las experiencias y discusiones de los feminismos sobre las TRHA resultan más recientes.

Respecto a los aspectos metodológicos, el análisis de las diferentes secciones es producto de una revisión de literatura enfocada en los debates de los feminismos sobre las TRHA. Se trabajó con fuentes secundarias, seleccionándose artículos y libros especializados en las perspectivas feministas sobre las TRHA, así mismo se complementó el análisis con fuentes audiovisuales.

Partiendo de las autoras referentes en las discusiones feministas sobre reproducción, se fueron reconstruyendo diferentes posturas tomando como primer criterio de búsqueda las referencias intertextuales entre las autoras en sus debates. Así mismo, la dimensión temporal determinó la búsqueda de fuentes y también constituye un organizador del artículo. Es por ello por lo que se intenta en su desarrollo respetar cierta genealogía de las discusiones, las llamadas «olas del feminismo», las cuales implicaron miradas y epistemologías diferenciales sobre la reproducción. Ello supone situarse en primer lugar en las autoras feministas europeas y norteamericanas, siendo Inglaterra el país pionero en su desarrollo, rápidamente sucedido por Estados Unidos y otros países de Europa. En este sentido, la dimensión geográfica es otro criterio presente, por lo que se partirá principalmente de las autoras feministas europeas y principalmente anglosajonas por ser estas últimas no sólo pioneras en su experiencia sino también promotoras de los primeros análisis sobre los desafíos de las TRHA para las mujeres.

\section{FEMINISMOS Y TRHA: UNA RELACIÓN «INCÓMODA».}

Desde el feminismo y los estudios de género, el debate sobre la maternidad, la reproducción y el control del sistema médico hegemónico sobre los cuerpos de las mujeres tiene un amplio recorrido desde diversas perspectivas 
feministas filosóficas, antropológicas y sociológicas. Desde el punto de vista de las mujeres y sus decisiones reproductivas, ha cuestionado históricamente el control del cuerpo de las mujeres así como la maternidad obligatoria. Podría decirse parafraseando a Irati Fernández Pujana que la relación entre la maternidad y el feminismo es una relación incómoda (149), tensión que se puede trasladar también a los estudios sobre TRHA, por reactualizarse algunas tensiones y preguntas sobre la maternidad como opresión patriarcal.

Si bien a continuación no se desarrollarán exhaustivamente las diferentes visiones del feminismo sobre la maternidad, estos debates atraviesan las tensiones de las posiciones feministas sobre TRHA. Una de las preguntas que atraviesa la teoría feminista se vincula a la autonomía sexual y reproductiva, específicamente si las tecnologías refuerzan la opresión patriarcal o posibilitan mayor autonomía reproductiva.

Para las feministas de la segunda ola, la maternidad constituía sin dudas una forma de opresión patriarcal, donde la experiencia de las mujeres indicaba que la decisión por la maternidad era muy restringida en algunos contextos y la sexualidad estaba confinada a la heterosexualidad, al matrimonio y donde la procreación era solo legítima en ese marco. Es por ello por lo que las luchas del feminismo estaban orientadas a la ruptura de la naturalización de la maternidad como destino para las mujeres en la sociedad (Chodorow 6; De Beauvoir 3).

Para Simone De Beauvoir, al igual que para otras feministas contemporáneas, el desafío estaba en disputar y deconstruir la idea del instinto materno por constituir una idea que sustentaba la opresión patriarcal hacia las mujeres (Saletti Cuesta 175). Enfatizando el papel de socialización patriarcal sobre la maternidad, la apuesta de Simone De Beauvoir se orientaba a la deconstrucción de la «mujer», como bien señala con la afirmación de que «mujer no se nace, se hace». Al mismo tiempo concebía los procesos reproductivos como una experiencia de alienación para las mujeres, que hacía sentir el cuerpo como algo ajeno «fatigoso» y que le exige a la mujer «pesados sacrificios» (De Beauvoir 12-13).

Así también las feministas de la segunda ola (Chodorow 11; De Beauvoir 12) cuestionaron la maternidad como identidad femenina. Cuando Nancy Chodorow se pregunta «¿Por qué las mujeres maternan?» analiza un conjunto de argumentos que históricamente han sustentado y naturalizado la

Feminismo/s 35, junio 2020, pp. 263-289 
división sexual del trabajo (11). Así también, otro aspecto central que plantea Chodorow es el cuestionamiento de la categoría «maternaje» ${ }^{1}$, noción que naturaliza el lugar de la mujer como cuidadora. De esta manera se produce, según la autora, la circularidad de la reproducción de género: «La división sexual del trabajo produce tanto las diferencias de género a la vez que es reproducida por ellas» (38). Para Chodorow, la división sexual no es solo un aprendizaje en el marco de la socialización de género, sino que forma parte de la conformación psíquica femenina en una sociedad patriarcal. Estas posiciones que cuestionan la maternidad como identidad femenina continúan presentes en desarrollos más recientes del feminismo francés, donde se critica la naturalidad del instinto maternal, las ambivalencias y opresiones en la experiencia de maternar (Badinter 19), entendiendo a las TRHA como nuevas formas de opresión sobre las mujeres (Laborie 5; Tubert, «Los ideales culturales» 161, «Demanda de hijo» 351).

Una posición que será controvertida en los años setenta es la de Shulamith Firestone, feminista radical, quien, al igual que De Beauvoir, planteaba que la maternidad era una experiencia victimizante para las mujeres (En Rich 174). Su postura es novedosa en cuanto ella consideraba que la tecnología podía contribuir a eliminar la diferencia sexual como origen de la opresión. La división sexual del trabajo y la tiranía de la familia biológica pueden resolverse con la reproducción artificial, decía Firestone como un planteamiento utópico (11). Desarrollos como el de Donna Haraway buscarán en la tecnología algo similar a lo que Firestone señalaba en la década de 1970 y que fue ampliamente criticado: la tecnología puede volverse una herramienta de liberación para las mujeres (Haraway, «Manifiesto para cyborgs» 7) (Franklin, «Transbiology» 19), idea que se retomará posteriormente ${ }^{2}$.

Una década después del planteo de Firestone y con el desarrollo de las TRHA, algunas feministas radicales como Gena Corea y Christine Crowe traerán estas discusiones para criticar las consecuencias de las tecnologías reproductivas para las mujeres. Crowe señala que las TRHA constituyen una

1. La autora señala que la categoría «paternaje» no existe, lo cual refleja esta naturalización de la división sexual del trabajo.

2. Puede rastrearse este paralelo teórico entre Firestone y Haraway en la conferencia brindada por Sarah Franklin en Barnard College. Consultar referencias. 
solución tecnológica para la ausencia de hijos pero que en lugar de apuntar a sus causas continúan reforzando la maternidad biológica como modelo deseable (547). La autora, en debate con aquellos argumentos que apuntan al derecho a decidir de las mujeres sobre su reproducción, cuestiona que la decisión no resulta autónoma en cuanto está mediada por circunstancias sociales como el estigma y la condena social por no tener hijos.

Otra exponente del feminismo radical, Janice Raymond, señala estas técnicas como una forma de violencia hacia las mujeres por degradar sus cuerpos (5) vinculándolas a formas de abuso sexual. Así también agrega la dimensión geopolítica trasnacional, analizando la gestación subrogada en países del sur global. En relación a las usuarias, Raymond también cuestiona la idea de elección de las mujeres sobre las TRHA, como valor que ha legitimado otras «opciones opresivas» tales como la prostitución y la pornografía. Al igual que Crowe, critica los argumentos que enfatizan la autonomía reproductiva de las mujeres, señalando que no puede haber elección si no hay autonomía, salud y justicia social (8). También critica cómo la reproducción asistida presenta al feto como un ente aislado e independiente del cuerpo de la mujer (9), la cual pasa a ser un mero ambiente y un envase, principalmente en la gestación subrogada.

Otro argumento crítico de la autora se vincula a la idea de que la infertilidad es producida por la reproducción tecnológica. En este sentido Janice Raymond argumenta que su creciente uso no solo se debe a que la definición de la infertilidad se ha expandido, sino a la oferta de especialistas y la consecuente visita a estos especialistas (39).

Desde el ecofeminismo Mies y Shiva señalan que las tecnologías de reproducción forman parte de los desarrollos y conquistas patriarcales y coloniales del hombre blanco sobre la naturaleza. Las autoras contrastan cómo las políticas de población intentan reducir los nacimientos en el «Tercer Mundo» mediante políticas de esterilización forzosa a las mujeres pobres, mientras que se busca la fertilidad en el «Primer Mundo». También subrayan una continuidad entre las políticas eugenésicas, el racismo y fascismo de la política nazi y las TRHA. Señalan que las tecnologías reproductivas también constituyen una política sexista, ya que permiten la selección del sexo, lo que tiene consecuencias graves particularmente en los cuerpos de las mujeres del «Tercer Mundo»(8). 
Desde otras posiciones políticas, algunas autoras contemporáneas sostienen críticas similares que van en la misma línea que el feminismo radical, particularmente la coincidencia se vincula a la relación entre las TRHA y el mercado reproductivo neoliberal y patriarcal. En la actualidad, algunas de estas críticas cuestionan la donación y mercantilización de los gametos y la gestación subrogada como otra práctica donde el escenario neoliberal da cuenta de un entramado del trabajo reproductivo realizado de manera desigual (Wichterich 17; Waldby y Cooper 21; Blázquez-Rodríguez 11). Estas autoras reconocen otras intersecciones vinculadas a la posición social, racial y territorial, que configuran diversos modos de desigualdad para las personas involucradas. Las autoras señalan que a nivel global es posible identificar una verdadera geopolítica de la mercantilización de la reproducción que reproduce las desigualdades globales, en consonancia con lo planteado por el feminismo radical en los años setenta. Aquí se denuncia que algunas sustancias biológicas tendrían más valor que otras (gametos de alta calidad y de baja calidad) vinculadas a color de piel, ojos y cabello, inteligencia, y educación, argumento vinculado a los debates sobre el racismo en la construcción del parentesco.

En sintonía con algunas críticas del feminismo radical, Sarah Franklin también cuestiona estas formas de trabajo reproductivo desigual y señala que uno de los grandes desafíos de las TRHA se remite a que nos encontramos con un campo desregulado, con las consecuencias que ello acarrea: la comercialización y la creación de un mercado reproductivo así como la perpetuación de las normas de género, raciales y de parentesco, mientras que ideas asociadas al fundacionalismo biológico están colapsando (8).

Así también se advierte que el contexto neoliberal posibilita y enfatiza la lógica de elección de las personas usuarias. Como señala Marylin Strathern, no es casual que esta idea de elección en el ámbito de la reproducción y el parentesco surja en Inglaterra en una sociedad capitalista donde priman una mirada individualista del ciudadano y una mirada emprendedurista del sujeto (Reproducing the future 38).

De esta manera, algunas de las críticas del feminismo radical, vinculadas a la conexión entre la mercantilización de la reproducción y el mercado neoliberal global y colonial, son compartidas por diferentes posiciones y continúan vigentes en los desarrollos posteriores. 
Es preciso también señalar otras dimensiones en las que es posible marcar algunas divisiones y fragmentaciones que se dieron dentro del feminismo tanto frente a algunas posturas críticas sobre las TRHA, como por plantear miradas divergentes sobre la maternidad.

Una de las feministas que presenta una postura diferente de la maternidad es Adrienne Rich, quien plantea que el problema no es la reproducción ni la maternidad en sí, sino la manera patriarcal en que se instituye la maternidad en nuestras sociedades (175). En sintonía con lo planteado por De Beauvoir, en estas circunstancias la maternidad alienaba a las mujeres y se convertía en un trabajo forzado. Sin embargo, diferenciaba esta opresión de la experiencia misma de maternar, entendiendo que podía convertirse en espacio de poder femenino más allá de las estructuras patriarcales. En esta misma línea, desde el feminismo de la diferencia como se plantea la importancia de recuperar el orden simbólico femenino, la genealogía femenina y las relaciones entre mujeres en una sociedad falogocéntrica (Irigaray 5; Saletti Cuesta 179)

En una lógica similar, es posible pensar algunas de estas tensiones y fragmentaciones del feminismo sobre la maternidad como en relación a las tecnologías reproductivas. Tanto Sarah Franklin como algunas autoras del feminismo radical como Gena Corea y Christine Crowe formaron parte de la red FINRRAGE ${ }^{3}$, el primer movimiento que dentro del feminismo planteó una postura crítica a las nuevas tecnologías reproductivas (Strathern, A Antropologia e o advento 9). Dentro del mismo se trazaron diferentes posiciones donde un sector disidente -que incluía a la ya mencionada Sarah Franklin- cuestionaba la mirada de un sector del feminismo radical, que entendía a las mujeres como víctimas, cómplices del sistema patriarcal o inocentes -en inglés dupes ${ }^{4}$ - frente a estas nuevas tecnologías (Franklin, The Politics of Reproduction). Esta forma de comprender la relación entre la autonomía de las mujeres y las tecnologías, provocó un conjunto de disputas internas que llevaron a la posterior fragmentación del movimiento (Franklin, «Transbiology» 2).

3. FINRRAGE: Feminist International Network of Resistance to Reproductive and Genetic Engineering

4. Hace referencia a la expresión de Renata Klein, la cual fue intensamente discutida en la organización. Para ver más información consultar la Conferencia brindada por Sarah Franklin, en Referencias.

Feminismo/s 35, junio 2020, pp. 263-289 
De esta manera, un primer punto en tensión que se puede señalar en el debate del feminismo sobre las TRHA, tuvo que ver con una concepción de sujeto y particularmente con la posibilidad de agencia de las mujeres frente a las tecnologías reproductivas. Estas posturas, sin desconocer algunas de las críticas planteadas por el feminismo radical, delimitaron otra relación posible con las tecnologías reproductivas desde una óptica que se desplazaba de la mirada de opresión, lo que suponía revisar la concepción de poder que sustentaba las otras posturas (Luna, Reproducción asistida 20).

Un aspecto relevante y novedoso de estas nuevas posiciones feministas parte del reconocimiento de aquello que Sarah Franklin denomina como una «incómoda ambivalencia» del feminismo frente a las TRHA y que suponía llevar adelante otra línea de acción. La autora señala que:

[...] paradójicamente contar con mayor información y mayor elección reproductiva podría ser opresivo y desempoderante. Esta ambivalencia no fue usada como base para rechazar la tecnología, sino para habilitar a las mujeres a negociar sus demandas de forma más efectiva» (Franklin, «Transbiology» 2) [la traducción es propia].

Siguiendo a Franklin, en lugar de rechazar las TRHA, el hecho de poder identificar las particulares maneras en que se desarrolla la opresión en el campo biomédico permitiría entonces pensar qué estrategias pueden fortalecer la autonomía reproductiva de las personas. Desde allí que las visiones de las usuarias de estas tecnologías fueran centrales para disputar estas relaciones de poder.

Es por ello por lo que un segundo punto en tensión con el feminismo radical se vincula a la crítica de que sus desarrollos teóricos se encuentran desconectados de las experiencias de las mujeres con las tecnologías reproductivas (Luna, Reproducción asistida 20). A partir de ello se advierte el desarrollo de estudios centrados en las experiencias concretas de las mujeres con las tecnologías, por ello algunas de estas autoras se involucraron con un estudio crítico del sistema de salud (Franklin, «Transbiology» 3).

De esta manera, el desarrollo de un movimiento por la salud de las mujeres en los años ochenta, conjuntamente con el desarrollo de estudios sobre diversas experiencias de mujeres con la tecnología reproductiva -de visualización, la amniocentesis y la hormonización- dieron lugar a un conjunto de análisis feministas sobre el sistema médico hegemónico y los efectos de la 
biomedicina sobre la reproducción de las mujeres. Entre ellos se encuentra una línea en análisis que desde el feminismo puso en cuestión el sistema médico hegemónico, como Robbie Davis-Floyd referente en los estudios sobre los procesos de medicalización de los nacimientos y el parto (294).

Entre los trabajos que desarrollaron estudios particulares sobre las TRHA en el campo biomédico, se destacan algunas autoras ya mencionadas como Sarah Franklin, Charis Thompson y Rayna Rapp. Otra autora que plantea un estudio empírico es Elaine Denny, quien también cuestiona la manera en que el feminismo radical ha utilizado la noción de elección de forma diferenciada, cuando se debate aborto o maternidad. Denny señala que siempre existe un conjunto de variables que en ambas opciones pueden mitigar la autonomía reproductiva de las mujeres y en este sentido entiende que el feminismo no debería oponerse a la tecnología sino a la manera en que está organizada (77).

La relevancia que estos aportes del feminismo tuvieron respecto al campo de estudio del cuerpo, la medicina y la reproducción, suelen ser desestimados desde la academia. Como señala Rayna Rapp y en coincidencia con otros análisis (Franklin, «Transbiology» 5; Tarducci 110), la antropología feminista desde los años setenta y aquellas inscritas dentro del movimiento de la salud de las mujeres de los ochenta, tuvieron un papel central al renovar el interés por la reproducción y el parentesco como aspectos centrales de debate en la academia que pocas veces es reconocido (Rapp, Gender, Body, Biomedicine 467). Otra área que se ve transformada en sus discusiones se vincula al estudio de los efectos de esta tecnología en la cultura y cómo permea en la construcción de las familias y las relaciones de parentesco (Yanagisako y Collier 34), donde las propuestas de las antropólogas feministas (Franklin, Embodied Progress; Strathern, After nature 35) renovaron los debates que se abrían en este campo antes dominado por el funcionalismo. Particularmente las TRHA y las modificaciones en el parentesco desafiaron las nociones de lo «lo social»y «lo natural» tan relevantes a la hora de la definición occidental sobre la familia (Luna, Pessoa e Parentesco 390) como del género (Stolke 79).

Dentro del campo de la antropología feminista, el trabajo de Rayna Rapp como el de Rosalind Petchensky fueron aportes centrales para dar algunas respuestas a esta «incómoda ambigüedad» frente a las TRHA (Franklin, «Transbiology» 5). Estas discusiones permitieron desarrollar dentro de los 
feminismos otras lecturas y posiciones diferentes donde la biotecnología dejo de ser vista como sinónimo de disciplinamiento del patriarcado.

Rosalind Petchesky, en su estudio sobre las tecnologías de visualización, señala esta misma tensión particularmente analizando el papel del ultrasonido. Si bien es crítica acerca de cómo las mismas refuerzan la subjetivización del feto, por presentarlo como un ente aislado, individual y funcional al control de los cuerpos de las mujeres, también señala la importancia de conocer las perspectivas de las mujeres ya que en general las mismas no se sienten ni víctimas ni pasivas frente a las tecnologías (279). En este sentido, se interroga sobre la tensión que se produce entre lo que el feminismo decodifica como opresión y las diversas experiencias de las usuarias. Petchensky también cuestiona la idea de que las mujeres usuarias de las tecnologías reproductivas reproducen de forma automática los modelos hegemónicos de familia heterosexual, conyugal, biológica (276).

Del mismo modo, Rayna Rapp es crítica sobre los efectos que han tenido las tecnologías de visualización, las cuales nacidas como una tecnología naval hoy resultan de uso cotidiano y tienen efectos en la relación médico-paciente y médico-mujeres: presenta la ilusión de un feto independiente, autónomo, descorporeizado de la mujer, donde el conocimiento y la interpretación de esta información es únicamente potestad médica. Sin embargo, cuando la autora estudia las decisiones de las mujeres frente a las biotecnologías, analiza las experiencias buscando comprender los obstáculos de las usuarias («Real-time fetus» 630). Partiendo de la decisión compleja que supone para las mujeres utilizar la amniocentesis ${ }^{5}$ durante el embarazo, Rayna Rapp utiliza el concepto de «pioneras morales» para hacer alusión a la manera en que las mujeres accionan en sus decisiones frente a este estudio prenatal (Testing Women 306).

Como se señaló anteriormente, estos desarrollos que dan cuenta del compromiso por comprender las experiencias de las mujeres con las tecnologías parten de y tienen un anclaje en su propia subjetividad: la autora comienza esta investigación a partir de su propia experiencia de aborto después de realizarse un estudio de amniocentesis, comprendiendo la complejidad de este escenario para las mujeres en el campo biomédico.

5. Punción mediante la cual se busca diagnosticar durante el embarazo, la presencia de una anomalía cromosómica o genética en el feto. 
Estos estudios, alejándose de miradas de la tecnología como necesariamente negativa, permiten comprender que las mujeres frente a estos nuevos desarrollos biomédicos comenzaron a encontrarse en situaciones de decisión en que las consecuencias de los tratamientos no se encontraban definidos de antemano. De esta manera, la autora entiende que esto lleva a que las personas como protagonistas de procesos reproductivos, sean cada vez más reflexivas. Como señala Rayna Rapp, existe una mayor autoconciencia de la manipulación de las categorías de parentesco y biología por parte de las personas usuarias, quienes transitan estos procesos como analistas de sus propias prácticas ( «Gender, Body, Biomedicine» 470).

Así también, lejos de evadir las relaciones de poder y desiguales en el campo biomédico, se propusieron nociones como la de «reproducción estratificada» (Colen 80) que permitieron comprender cómo ciertos desarrollos biomédicos reforzaron la patologización de la personalidad, el género y el parentesco (Rapp, «Gender, Body, Biomedicine» 469). Esta reproducción ahora entendida como problemática y patológica es la que posibilita la mercantilización de la reproducción con la vigilancia del estado (Rapp, «Gender, Body, Biomedicine» 469). Sin embargo, estas críticas no desconocen la ironía en estos procesos: a la vez que patologizan y estigmatizan ciertas formas de reproducción, dan lugar a la conformación de nuevas políticas identitarias (Rapp, «Gender, Body, Biomedicine» 469).

Volviendo a la tensión planteada por las autoras, no se puede desconocer cómo las TRHA han abierto nuevas opciones de elección, las cuales tienen un costo que se vincula a la creciente medicalización de los cuerpos, de la sexualidad y a la responsabilización de las mujeres por la gestión «eficiente» de su reproducción. A su vez, esta tensión entre elección-responsabilización reformula la pregunta por el poder y la agencia de las mujeres en relación a estas técnicas.

A continuación se planteará un conjunto de posturas divergentes dentro del feminismo que no solo repercutirán en la manera en que se entiende la tecnología reproductiva, sino que propone desde el feminismo otra manera de vincularse con la «naturaleza» y la «tecnología», o más bien de deconstruir esta falsa oposición. 


\section{FEMINISMOS, PODER Y TECNOLOGÍA ¿OTRA RELACIÓN ES POSIBLE?}

Al mismo tiempo que algunos sectores del feminismo radical en la década de los noventa reafirmaban su oposición a las TRHA, desde otros sectores de feminismo se comenzó a pensar de otra manera las relaciones entre tecnología y reproducción, donde el cuerpo constituyó un eje importante para entender las relaciones de poder.

Al igual que Firestone, quien en la década de los setenta pensaba que la tecnología permitiría romper con la opresión de la diferencia sexual, algunas feministas como Donna Haraway propusieron nuevas formas de alianzas utópicas entre feminismo y tecnología («Las promesas» 141). Sin desconocer las relaciones de poder patriarcales y neoliberales, estas nuevas posiciones comenzaron a cuestionar cierta relación lineal donde la tecnología es entendida como villana y las mujeres se encuentran desagenciadas (Franklin, «Transbiology» 3; Haraway, «Manifiesto para cyborgs» 7; Preciado, Testo Yonqui 40; Rapp, Testing Women 86).

Como señala Sarah Franklin, la pregunta por la relación entre la reproducción y la tecnología es una pregunta por el poder. Es por ello por lo que las posiciones presentadas previamente han permitido pensar desde otra óptica otras relaciones de poder entre las mujeres, su reproducción y la tecnología (7).

Si hay una única lección para llevar de este cuerpo entero de académicas feministas, es que la relación entre tecnología y reproducción nunca puede separarse de cuestiones más amplias sobre el estatus y el empoderamiento de las mujeres. En el borroso margen entre las opciones limitadas y el hecho de no-tener-más-opción-que-elegir-entre-ellas se encuentra la paradoja característica del debate feminista sobre las nuevas tecnologías reproductivas (Franklin, «Transbiology» 2) [la traducción es propia y el énfasis original de la autora]

Una dimensión donde se ancla esta mirada del poder y se piensa la autonomía reproductiva es la dimensión corporal, la cual puede ser vista como plataforma de control pero también como un eje de resistencia y transformación. Aquí el feminismo y los estudios queer traerán discusiones críticas sobre la existencia de la biología, la naturaleza y la noción de «sexo» como base material. La radicalidad de estos debates no es menor, dado que plantean que no 
hay una «vuelta atrás» hacia la naturaleza para pensar la reproducción. Como señala Donna Haraway, desde estas perspectivas la naturaleza es discursiva, es un lugar retórico común pero ya no entendida como aquel lugar estable de certezas, sino que es el lugar del cambio:

La naturaleza es también un trópos, un tropo. Es figura, construcción, artefacto, movimiento, desplazamiento. La naturaleza no puede preexistir a su construcción. Esta construcción se articula sobre un determinado movimiento, un tropos o «giro». Fieles a los griegos, en tanto que trópos, la naturaleza tiene que ver con cambiar. (Haraway, «Las Promesas» 122-123)

Esto supone, a diferencia de las primeras posturas feministas analizadas, que la construcción de una mirada crítica y feminista sobre la biología constituye un giro radical, entendiéndola como un artefacto y un discurso sustentado por el aparato científico. Ello modifica la forma de comprender los cuerpos de las personas gestantes, los fetos y los embriones, ya no como un ente natural diferente a la tecnología, sino como parte de la misma.

En esta línea de análisis que incorpora los efectos de la biomedicina sobre los cuerpos, se puede señalar el trabajo de Emily Martin, quien puso de relieve la construcción socio política y patriarcal de la sexualidad y la reproducción (45). Ello le permitió a la autora advertir en los discursos médicos la reproducción de representaciones de género sobre los cuerpos femeninos, discursos que eran reproducidos por las mismas mujeres. Así también su trabajo señala la sensación de enajenación de las mujeres sobre el propio cuerpo, percibiéndolo como un ente-objeto que es animado pero operado por otros, como una máquina, en un proceso de alienación que De Beauvoir ya señalaba décadas atrás.

Si con Emily Martin podemos pensar en el cuerpo como máquina (61), con Haraway hablamos del cuerpo como sistema ( Manifiesto para cyborgs» 7), metáforas que no sólo expresan posiciones filosóficas sobre el cuerpo, sino que tienen consecuencias políticas sobre la capacidad de agencia. Haraway a su vez propone la idea de cyborg como una construcción hibrida, de organismo y máquina, donde las tecnologías y sistemas de comunicación constituyen un lugar a disputar («Las promesas» 2). Las ideas de cyborg de Haraway y de una transbiología son nociones que permiten romper con visiones generizadas de la biología, a la vez que proyectar irónicamente otras formas de convivencia 
de la biología y la tecnología, que no son parte de lo natural, sino metáforas que permiten pensar su transformación (Franklin, «Transbiology» 7).

Donna Haraway, dialogando con Sarah Franklin, cuestiona ese lugar de apropiación de aquello construido discursivamente como naturaleza, donde la gestión del feto es un ejemplo que da cuenta de lo que es naturaleza y humano, constituyendo uno de los planos -interno le llama la autora- que el sistema científico capitalista busca controlar («Las promesas» 146). Sarah Franklin en la misma línea analiza las nuevas formas de control y de gestión de la biología, cuyo propósito está puesto en hacerla más confiable a través del «control del riesgo» de estos procesos («The Cyborg Embryo»178).

Marilyn Strathern por su parte señala que si bien las nociones de cuerpo y máquina fueron conectadas por analogía, ya no funcionan más en los sentidos que se le asigna a la tecnología actualmente. Por el contrario, como sucede en el caso de las TRHA, se entiende que la tecnología ayuda a la vida a funcionar, en el sentido cultural de «asistir a la naturaleza» (Reproducing the future 60). Por ello, desde la visión de Strathern, naturaleza y tecnología no evocan distintos dominios de la vida, por lo que no sirven de metáfora la una a la otra (Reproducing the future 60).

Otro aspecto que constituye una visión divergente dentro del feminismo para pensar las tecnologías reproductivas se vincula a la manera en que se piensa el poder y la agencia desde concepciones micropolíticas. Tal es el caso de Teresa De Lauretis, quien, siguiendo a Foucault, desarrolla la noción de «tecnologías de género». Para ella, la resistencia al poder y la agencia se pueden comprender desde los intersticios del poder (De Lauretis 33) y no desde el planteamiento clásico de resistencia.

Paul Preciado, como referente de los estudios queer, parte de una mirada del cuerpo que pone en cuestión la tradicional mirada del feminismo que sitúa en el sexo como la naturaleza o base material y el género como la cultura o la interpretación de esa naturaleza. La ruptura de este binarismo le permite comprender una idea maleable del cuerpo que puede ser transformado desde las propias tecnologías médicas como la hormonización. Para Preciado, el «tecnobiopoder» opera a nivel micro y forma parte de las subjetividades de tal manera que este poder es voluntariamente inserto en el cuerpo, como sucede en la autoadministración de hormonas (Testo Yonqui 40). La propia 
experiencia de autoadministración de la testosterona da cuenta de otros procesos impensados como la apropiación de la biotecnología por parte de los sujetos.

El planteamiento de Paul Preciado, en línea con lo que expresa Donna Haraway, abre otro juego para pensar la relación con la tecnología y permite entender a los cuerpos de las personas usuarias no como un ente previo, natural y diferente a la tecnología, sino como un «tecnocuerpo» que no es «ni organismo ni máquina» (Preciado, Testo Yonqui 40) proyectando de esta manera otro orden de relaciones y diferentes a las primeras posiciones del feminismo.

La perspectiva queer permite cuestionar en la actualidad no solo los sentidos sobre cuerpo y tecnología, sino también la construcción social y política de la reproducción. Como advierte Preciado, es el mismo Estado quien define quiénes pueden acceder a las TRHA, donde la heterosexualidad continúa siendo el régimen político y la tecnología social con los que se piensa la reproducción («Procreación» 67). De esta manera, esa perspectiva sitúa el acceso a las TRHA como una disputa política y un proceso de despatologización de aquellas personas que han sido privadas históricamente y políticamente de su reproducción. Sin embargo, es interesante cómo Preciado, tomando los aportes de Silvia Federici, señala cómo el útero continúa siendo central en el proceso de acumulación capitalista y colonial («Procreación» 74).

En el marco de la reconstrucción de estos debates, es interesante pensar que estas tensiones hasta aquí analizadas dan cuenta de que estas fracciones políticas son contextuales, donde los planteamientos de las feministas radicales que denunciaban la mercantilización y colonialidad de la biotecnología aún resuenan en los desafíos y disputas actuales por la regulación de las tecnologías reproductivas a nivel global, como analizaremos a continuación.

\section{FEMINISMOS Y TRHA EN LATIONAMÉRICA Y ARGENTINA: DEMANDAS REPRODUCTIVAS ACTUALES.}

En Latinoamérica son recientes las producciones que desde el feminismo han analizado las TRHA y están directamente vinculadas a la agenda política y la discusión pública. Como señalan Cardaci y Sánchez Bringas para el caso mexicano, más allá de algunas denuncias realizadas desde los feminismos contra 
estas técnicas como nuevas formas de opresión, han permanecido más bien ajenos a estos debates y tampoco han realizado investigaciones sistemáticas al respecto, a excepción de algunas autoras (271).

El caso brasilero ha retomado estos debates en su producción por ser relevante a nivel de las experiencias: es el país latinoamericano donde se realizan más tratamientos ${ }^{6}$. Sin embargo no es la opresión o la autonomía su principal eje de análisis, sino que sus trabajos se han centrado en las transformaciones sobre el género y el parentesco (Fonseca 781; Grossi, Uziel y Mello 9; Luna, Pessoa e Parentesco 389; Ramírez-Gálvez 4) dialogando con los debates del norte global sobre las TRHA. Así también se destacan trabajos de Uruguay (Viera Cherro 351) como investigaciones situadas en Ecuador de corte antropológico (Roberts 15).

Particularmente en Argentina, país en el que se centra este trabajo, existe una regulación desde el año 2013 que habilita el acceso de forma universal a las TRHA y define su cobertura, pero aún quedan diversos vacíos por regulación: la gestación subrogada, el tratamiento de los embriones in vitro y la regulación de la donación de gametos. En estos temas el feminismo no constituye la voz más visible, sino que son abordados a «nivel de expertos» (Fraser 8).

Por este motivo, los debates en Argentina no transcurren sobre los efectos de los cuerpos de las mujeres como principal eje, sino sobre cómo las mismas «funcionan» y cómo deben ser reguladas. De esta manera se encuentran algunos trabajos que desde las ciencias sociales incorporan la perspectiva de género (Garay 30) pero principalmente se encuentran trabajos desde la sociología (Ariza, «La construcción» 43; Straw, Scardino, y Pérez 3) y los estudios de la ciencia y la tecnología centrados en estos temas (Ariza, «Cuerpos abstractos» 363) analizando el papel de diferentes actores en su regulación (Ariza, «La regulación» 8; Farji Neer 351; Irrazábal 113). Algunas autoras parten desde enfoques etnográficos sobre la experiencia de donantes de gametos (Ariza, «No pagaras» 240), sin perder de vista las condiciones sociales

6. De acuerdo con un informe de la RED LARA (Registro Latinoamericano de Reproducción Asistida), Brasil es el país latinoamericano con mayor cantidad procedimientos de reproducción asistida realizados en ese año, seguido por Argentina y luego México. Ver referencias Zegers-Hochschild, Schwarze, Musri, Crosby y Urbina. 
y globales en que se desarrolla este trabajo reproductivo pero aportando a comprender este escenario desregulado.

Fundamentalmente en Argentina la demanda por la legalización del aborto, como un tema de debate social y fuerte impacto en la opinión pública, aparece de forma espejada frente a este vacío normativo respecto al tratamiento del embrión no implantado, aspecto que es analizado principalmente desde la sociología y las ciencias jurídicas (Jadur, Duhalde y Wainstein 33; Lamm 2; Straw, Scardino y Perez 3). En este sentido, desde los feminismos en Argentina se encuentran análisis acerca de los discursos conservadores sobre el embrión, politizando las consecuencias que tienen en la criminalización del aborto (Morán Faúndes 151; Vacarezza 71) pero sin abordar los efectos sobre la misma practica de las TRHA.

Por todo ello, las voces más presentes en Argentina sobre las TRHA son las disciplinas jurídicas, que retoman discusiones del feminismo pero se centran más bien en los derechos reproductivos y la discusión por las nuevas formas de filiación (Herrera y Lamm 5; Lamm 3) y la gestación subrogada.

Los términos en que se plantea la discusión no se vinculan a la maternidad, como pasaba en los primeros debates, sino que el tema que polariza posiciones en los feminismos latinoamericanos es el debate «trabajo sexual versus prostitución». La gestación subrogada es una práctica sobre la que no existe consenso en los feminismos a nivel global ni regional. Como señala Olavarría para el caso mexicano, tanto legisladores, juristas como activistas buscan «puntos de intersección o modelos que permitan su comprensión» (3) ante un tema que es comparado con otras prácticas presentes en el imaginario social, tales como la figura de la «gestante madre», «gestante donante», «gestante cuidadora»y «gestante prostituta». Estos paralelos se establecen ya que todos los debates ponen en juego la pregunta por la autonomía corporal de las mujeres en un mercado reproductivo y sexual global atravesado por formas de «reproducción estratificada» (Colen 82; Rapp, «Gender, Body, Biomedicine» 469).

Esta noción resulta clara para comprender las jerarquizaciones que atraviesan «todas las formas posibles de convertirse en padres, ya sea a través de la procreación, la adopción o la reproducción médicamente asistida, así como el ejercicio mismo de la parentalidad» (Olavarría 16). Pero así también esta estratificación da cuenta de diferentes formas de care o de cuidados, donde la

Feminismo/s 35, junio 2020, pp. 263-289 
gestación constituye el último escalón en esta estratificación de tareas reproductivas que no son reconocidas generalmente como un trabajo.

Particularmente en Argentina los sectores que defienden su regulación están confirmados por colectivos juristas especializadas en el tema (González Magaña 5; Herrera y Lamm 5), en alianza con colectivos biomédicos y organizaciones de personas usuarias y de diversidad sexual (Rodríguez Iturburu y Notrica 2$)^{7}$. El último proyecto legislativo supone reconocer la existencia de una práctica que ya existe en Argentina junto con el reconocimiento de la identidad de las personas nacidas de la misma; pero al mismo tiempo busca evitar condiciones que habiliten la creación de un mercado reproductivo como en otros países donde ocurre a niveles de explotación.

Sin embargo, desde los feminismos y la academia también existen posturas críticas a esta técnica y que denuncian este trabajo reproductivo como opresivo, como es el caso de Dora Barrancos, quien cuestiona esta práctica por su carácter eugenésico y racista. La autora, sin embargo, diferencia el trabajo sexual y reproductivo de la práctica de la gestación subrogada porque no implica a una tercera persona, así como también lo diferencia del aborto porque lo entiende como una demanda que se basa en la dignidad del propio cuerpo, aspecto que en la gestación subrogada se vulnera. A diferencia de las juristas argentinas, Barrancos sostiene que no es argumento suficiente que una práctica exista para que deba ser regulada (156). Como se anticipó, lo que sucede en este escenario es que el eje de fractura está dado por la pregunta por la regulación/prohibición como estrategia política de incidencia sobre la reproducción.

\section{RECAPITULANDO UN DEBATE INCONCLUSO}

A modo de cierre, y retomando la experiencia que nos dejan las primeras posiciones del feminismo sobre las TRHA, se pueden revisar algunos caminos ya transitados para continuar repensando el aporte teórico y político del feminismo. Esta reconstrucción realizada permite advertir que ciertas tensiones, en apariencia irreconciliables en los primeros debates, hoy se amalgaman de diferentes formas a la luz de los desafíos actuales. Así mismo permite

7. En Argentina existen dos proyectos de Ley que buscan su regulación, pero con diferentes énfasis e impulsados por distintos actores.

Feminismo/s 35, junio 2020, pp. 263-289 
reconocer que los debates sobre las THRA visibilizan el trabajo reproductivo, históricamente naturalizado y estratificado, que hoy se identifica en las diversas prácticas de donar gametos, gestar, cuidar y criar. Sin embargo, las formas de explotación del cuidado también se reinventan en diferentes momentos históricos y mediante otras tecnologías políticas, si pensamos en el trabajo de nodrizas, amas de leche, ejerciendo la crianza y el cuidado de sectores acomodados. Estos aspectos que se producen de forma disociada en las diferentes conformaciones familiares y de cuidado, dan cuenta de condiciones de explotación desigual en diversas sociedades, más allá de la existencia de las TRHA.

Así también, esta reconstrucción nos señala la importancia de atender a las experiencias situadas que las TRHA generan en tanto opresión como posibilidad de agencia, para evitar caer en sentidos universalizantes sobre la experiencia «femenina» que tan cuestionados han sido dentro del pensamiento feminista.

Atender a la experiencia de las personas no nos debe distraer de un mapa mayor del cual las feministas radicales advirtieron desde un primer momento: el análisis de estas experiencias no es posible si no se tiene en cuenta la división del trabajo sexual, reproductivo y de cuidados a nivel global, para develar estratificaciones reproductivas que operan en diferentes espacios sociales.

Desde los feminismos latinoamericanos es posible plantearse otras disputas epistemológicas. La mirada que proponen las epistemologías del sur resulta una intersección de poder poco explorada para pensar estas nuevas formas en que se produce la reproducción. Siguiendo a Andrea Torrano y Natalia Fischetti, es posible pensar las tecnologías desde el «Buen Vivir», entendiendo que existe «una relación co-constitutiva entre lo humano, lo técnico y lo tecnológico y la naturaleza» (275), miradas que alientan a no reproducir lógicas extractivistas sobre los cuerpos que gestan y llevan adelante el cuidado.

A partir de estos debates, los feminismos nos permiten responder a la pregunta por las TRHA y el poder, con otras herramientas y nuevas preguntas. Por un lado, se pueden pensar las TRHA desde los feminismos sin recurrir a la sacralización de la maternidad ni la naturaleza. Así mismo nos interpelan cuando dan voz a las experiencias situadas y pueden fortalecer las formas de resistir y construir agencia que las/os sujetas/s encuentran en los lugares más 
inesperados. Sin embargo, no podemos perder de vista que estos procesos se producen en un escenario capitalista, patriarcal y colonial que se reinventa y donde no existen respuestas definitivas sobre la tecnología como dispositivo social y político, dejándonos en una constante incomodidad y búsqueda.

\section{REFERENCIAS BIBLIOGRÁFICAS}

Ariza, Lucía. «La construcción narrativa de la infertilidad. Mujeres que narran la experiencia de no poder concebir». Sexualidad, Salud y Sociedad. Revista Latinoamericana 18 (2014): 41-73.

Ariza, Lucía. «Cuerpos abstractos, riesgos concretos: Dispositivos clínicos y la salud de las donantes de óvulos en la medicina reproductiva Argentina». Salud Colectiva 12.3 (2016): 361-382.

Ariza, Lucía. «'No pagarás': el Consentimiento Informado como productor de solidaridad en la medicina reproductiva». Ciencia, Docencia y Tecnología 27.52 (2016): 240-268.

Ariza, Lucía. «La regulación de las tecnologías reproductivas y genéticas en Argentina: análisis del debate parlamentario». Dossiêr: Conservadorismo, Direitos, Moralidades e Violência 50 (2017): e175005.

Armstrong, David. «The rise of surveillance medicine». Sociology of Health $\mathcal{E}$ Illness 17.3 (1995): 393-404.

Badinter, Elizabeth. La mujer y la madre. Un libro polémico sobre la maternidad como nueva forma de esclavitud. Madrid: La esfera, 2012.

Barrancos, Dora. «Dilemas éticos de la reproducción tecno-mediada: una reflexión más allá de la cosmovisión religiosa». Sociedad y Religión: Sociología, Antropología e Historia de la Religión en el Cono Sur 25.44 (2015): 155-179.

Blázquez-Rodríguez, María Isabel. «Aproximación a La Antropología De La Reproducción». AIBR. Revista de Antropología Iberoamericana 42 (2005): 1-25.

Cardaci, Dora, y Ángeles Sánchez Bringas. «Hasta que lo alcancemos... Producción académica sobre reproducción asistida en publicaciones mexicanas». Alteridades 19.38 (2009): 21-40.

Chodorow, Nancy. The Reproduction of Mothering. Psychoanalysis and the Sociology of Gender. Berkeley, Los Angeles: University of California Press, 1978.

Colen, Shelee. «Like a mother to them». Conceiving the new world order. The global politics of reproduction. Eds. Faye D. Gynsburg y Rayna Rapp. California: University California Press, 1995. 78-102. 
Corea, Gena. «The Mother Machine: reproductive technologies from Artificial Insemination to Artificial Wombs». The American Journal of Maternal/Child Nursing 32.1 (1985): 357-363.

Crowe, Cristine. ««Women want it»: In-vitro fertilization and women's motivations for participation.» Women's Studies International Forum 8.6 (1985): 547-552.

Davis-Floyd, Robbie. "Obstetric Training as a Rite of Passage». Obstetrics in the United States: Woman, Physician, and Society 1. 3 (1987): 288-318.

De Beauvoir, Simone. El segundo sexo. Los hechos y los mitos. La experiencia vivida. Buenos Aires: Siglo XXI, 1949.

Denny, Elaine. «Liberation or oppression? Radical feminism and in vitro fertilization». Sociology of Health E Illness 16.1 (1994): 62-80.

De Lauretis, Teresa. «La tecnología del género». Revista Mora 2 (1996): 6-34.

Farji Neer, Anahí. «Cuerpo, derechos y salud integral: Análisis de los debates parlamentarios de las leyes de Identidad de Género y Fertilización Asistida (Argentina, 2011-2013)». Salud Colectiva 11. 3 (2015): 351-365.

Fernández Pujana, Irati.«Feminismo y maternidad: ¿una relación incómoda? Conciencia y estrategias emocionales de mujeres feministas en sus experiencias de maternidad.». País Vasco: Emakunde/Instituto Vasco de la Mujer, 2014.

Firestone, Shulamith. The dialectic of sex: The case for feminist revolution. New York: Farrar, Straus and Giroux, 2003.

Fonseca, Claudia. «Homoparentalidade: novas luzes sobre o parentesco». Revista Estudos Feministas 16. 3 (2008): 769-783.

Foucault, Michel. Historia de la sexualidad. La voluntad de saber. Buenos Aires: Editorial Siglo XXI, 2008.

Franklin, Sarah. Embodied Progress. London: Routledge, 1997.

Franklin, Sarah. «The Cyborg Embryo: Our Path to Transbiology». Theory, Culture E Society 23. 7-8 (2006): 167-187.

Franklin, Sarah. «Transbiology: A Feminist Cultural Account of Being After IVF». Critical Conceptions: Techonology, Justice, and the Global Reproductive Market. New York: Bernard Center for Research on Women, 2009.

Franklin, Sarah. The Politics of Reproduction: New Technologies of Life. Conferencia brindada en Barnard College en ocasión de The Scholar \& Feminist Conference, 2009. 25 marzo 2020. https://vimeo.com/4635010. 
Fraser, Nancy. «La lucha por las necesidades: Esbozo de una teoría crítica socialista-feminista de la cultura política del capitalismo tardío». Debate feminista 3 (1991): 3-40.

Garay, Ricardo. «El destino de ser madres: la ideología de la maternidad como soporte discursivo de las nuevas tecnologías reproductivas». Maternidades en el siglo XXI. Ed. Mónica Tarducci. Buenos Aires: Editorial Espacio, 2008. 29-59.

González Magaña, Ignacio. «La tácita inclusión de la gestación por sustitución en el nuevo Código Civil y Comercial de la Nación. Preámbulo necesario de una norma expresa que la regule». La Ley 181 (2014): AR/DOC/3853/2014. 13 abril 2020.

Grossi, Miriam, Anna Paula Uziel, y Luiz Mello. Conjugalidades, parentalidades e identidades lésbicas, gays e travestis. Río de Janeiro: Garamond, 2012.

Haraway, Donna. «Manifiesto para cyborgs: ciencia, tecnología y feminismo socialista a finales del siglo XX». Ciencia, Cyborgs y Mujeres: La Reivindicación de La Naturaleza. Madrid: Ediciones Cátedra, 1995. 251-311.

Haraway, Donna. «Las promesas de los monstruos: Una política regeneradora para otros inapropiados/bles». Política y Sociedad 30 (1999): 121-164.

Herrera, Marisa, y Elenora Lamm. «Un valiente fallo del TEDH sobre gestación por sustitución. Prohibir, silenciar, regular o fallar». La Ley 122 (2014): 1-8. Irigaray, Lucy. An Ethics of Sexual Difference. New York: Cornell University Press, 1993.

Irrazábal, Gabriela. «Bioeticistas católicos en contra de las técnicas de reproducción asistida. Implicancias para la futura Reforma del Código Civil». Revista de Derecho de Familia 57 (2012): 113-134.

Jadur, Silvia, Constanza. Duhalde, y Viviana Wainstein. «Efectos emocionales de la criopreservación de embriones y su transferencia». Reproducción 25 (2010): 33-39.

Laborie, Françoise. «New reproductive technologies: news from France and elsewhere». Reproductive and Genetic Engineering 1.1 (1988): 77-85.

Lamm, Eleonora. «El status del embrión in vitro y su impacto en las técnicas de reproducción humana asistida. Aclarando conceptos para garantizar derechos humanos». La Ley 43 (2015): AR/DOC/1297/2015. 13 abril 2020.

Luna, Florencia. Reproducción asistida, género y derechos humanos en América Latina. San José: Instituto Interamericano de Derechos Humanos, 2008. 
Luna, Naara. «Pessoa e parentesco nas novas tecnologias reprodutivas». Revista Estudos Feministas 9. 2 (2001): 389-413.

Martin, Emily. The Woman in the Body. A Cultural Analysis of Reproduction. Boston: Beacon Press, 2001.

Mies, Maria, y Vandana Shiva. La Praxis Del Ecofeminismo. Biotecnología Consumo y Reproducción. Barcelona: Icaria Antrazyt. Mujeres, voces y propuestas, 1998. Morán Faúndes, José Manuel. «La valoración de la vida, la subjetivación del embrión y el debate sobre el aborto: aportes desde una perspectiva crítica». Acta bioethica 20. 2 (2014): 151-157.

Olavarría, María Eugenia. «La gestante sustituta en México y la noción de trabajo reproductivo». Revista Interdisciplinaria de Estudios de Género de El Colegio de México 4 (2018).

Petchesky, Rosalind. «Fetal Images: The Power of Visual Culture in the Politics of Reproduction». Feminist studies 13. 2 (1987): 263-292.

Preciado, Paul B. Testo Yonqui. Madrid: Editorial Calpe, 2008.

Preciado, Paul B. «Procreación políticamente asistida». Parole de queer (2014). 8 marzo 2020.

Ramírez-Gálvez, Marta Celia. Novas Tecnologias Reprodutivas conceptivas: fabricando a vida, fabricando o futuro. Diss. Universidade Estadual de Campinas, 2003.

Rapp, Rayna. Testing Women, Testing the Fetus: The Social Impact of Amniocentesis in America (Anthropology of Everyday Life). New York: Routledge, 1999.

Rapp, Rayna. «Gender, Body, Biomedicine: How Some Feminist Concerns Dragged Reproduction to the Center of Social Theory». Medical Anthropology Quarterly 15.4 (2001): 466-477.

Rapp, Rayna. «Real-time fetus: the role of the sonogram in the age of monitored reproduction». Beyond the body proper: Reading the Antropology of Material Life. Eds. Margaret Lock y Judith Farquhar. Durham, London: Duke University, 2007. 608-622.

Raymond, Janice. Women as Wombs. Reproductive Technologies and the Battle Over Women's Freedom. Melbourne: Spinifex Press, 1994.

Rich, Adrianne. Of Woman Born: Motherhood as Experience and Institution. New York: WW Norton, 1995.

Roberts, Elizabeth. God's laboratory: assisted reproduction in the Andes. California: Berkeley, 2012. 
Rodríguez Iturburu, Mariana, y Federico Notrica. «La figura de la gestación por sustitución, otra vez en boca de todxs». Microjuris (2017). 31 marzo 2020.

Rose, Nikolas. The Politics of Life Itself. Biomedicine, Power and Subjetivity in the Twenty First Century. New Jersey: Princeton, 2007.

Saletti Cuesta, Lorena. «Propuestas teóricas feministas en relación al concepto de maternidad». Clepsydra. Revista de Estudios de Género y Feminista 7 (2008): 169-183.

Stolke, Verena. «La mujer es puro cuento: la cultura del género». Estudos feministas, Florianápolis 12. 2 (2004): 77-105.

Strathern, Marylin. After nature: English kinship in the late twentieth century. Cambridge University Press: Lewis Henry Morgan Lectures 1989, 1992.

Strathern, Marylin. Reproducing the future. Antropology, Kinship, New Reproductive Technologies. Manchester: Manchester University Press,1992.

Strathern, Marylin. «A Antropologia e o advento da Fertilização In Vitro no Reino Unido: uma história curta». Cadernos Pagu 33 (2009): 9-55.

Straw, Cecilia, Marisa Scardino, y Agustina Pérez. «La mirada de usuarios de técnicas de reproducción asistida con donante o gestante en relación con los embriones». Rev. IUS 11.39 (2017): 1-26.

Tarducci, Mónica. «Adopción y parentesco desde la antropología feminista». Revista de Estudios de Género. La Ventana 4.37 (2013): 106-145.

Thompson, Charis. Making parents. The Ontological Choreography of Reproductive Technologies. Cambridge. London: MIT Press books, 2005.

Torrano, Andrea y Natalia Fischetti. «Apuestas del feminismo: Ciencia/ Técnica/ Latinoamérica. Nuevas urdimbres desde el Sur. (Hacia un buen vivir feminista)». RevIISE - Revista De Ciencias Sociales Y Humanas 11.11 (2018): 267-279.

Tubert, Silvia. «Demanda de hijo y deseo de ser madre». Debate Feminista 8 (1993): 349-377.

Tubert, Silvia. «Los ideales culturales de la feminidad y sus efectos sobre el cuerpo de las mujeres». Quaderns de Psicologia 12. 2 (2010): 161-174.

Vacarezza, Nayla. «Los fetos de otra manera. Reflexiones sobre afectos, aborto y políticas reproductivas a partir de obras de Zaida González y Felipe Rivas San Martín». Afectos políticos. Eds. D. Losiggio y C. Macón. Buenos Aires: Ensayos sobre actualidad, 2017. 71-91. 
Viera Cherro, Mariana. «Sujetos y cuerpos asistidos: un análisis de la reproducción asistida en el río de la plata». Civitas-Revista de Ciências Sociais 15. 2 (2015): 350-368.

Waldby, Catherine, y Melinda Cooper. «The Biopolitics of Reproduction: PostFordist Biotechnology and Women's Clinical Labour». Australian Feminist Studies 23. 55 (2008): 57-73.

Wichterich, Chista. Sexual and Reproductive Rights. Vol.11. Berlin: Heinrich Böll Foundation, 2014.

Yanagisako, Silvia y Jane Collier. «Género y Parentesco Reconsiderados: Hacia un Análisis Unificado». Assessing Cultural Anthropology. Ed. Robert Borofsky. Honolulu: Hawaii Pacific University, 1994.

Zegers-Hochschild, Fernando, Juan Enrique Schwarze, Javier Musri, Carolina Crosby, y María Teresa Urbina, «Assisted Reproductive Techniques in Latin America: The Latin American Registry 2015». JBRA Assisted Reproduction 23. 2 (2018): 143-153. 